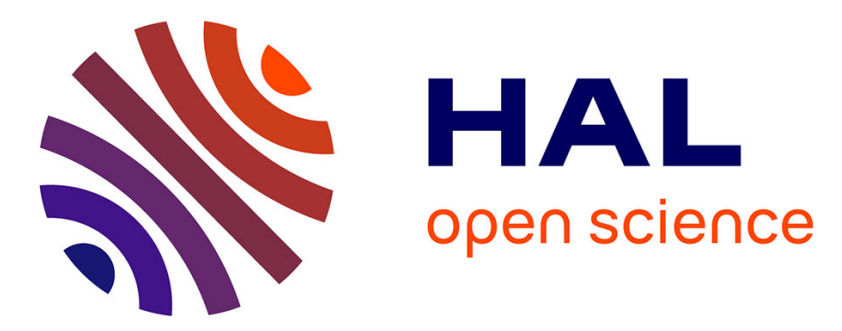

\title{
Patterned L10-FePt for polarization of magnetic films
}

Yannick Dusch, Nicolas Tiercelin, A. Klimov, V. Rudenko, Y. Ignatov, S.

Hage-Ali, Philippe Pernod, Vladimir Preobrazhensky

\section{To cite this version:}

Yannick Dusch, Nicolas Tiercelin, A. Klimov, V. Rudenko, Y. Ignatov, et al.. Patterned L10FePt for polarization of magnetic films. Journal of Applied Physics, 2011, 109, pp.07A720-1-3. 10.1063/1.3561172 . hal-00591347

\section{HAL Id: hal-00591347 \\ https://hal.science/hal-00591347}

Submitted on 4 Jun 2020

HAL is a multi-disciplinary open access archive for the deposit and dissemination of scientific research documents, whether they are published or not. The documents may come from teaching and research institutions in France or abroad, or from public or private research centers.
L'archive ouverte pluridisciplinaire HAL, est destinée au dépôt et à la diffusion de documents scientifiques de niveau recherche, publiés ou non, émanant des établissements d'enseignement et de recherche français ou étrangers, des laboratoires publics ou privés. 


\section{Patterned $L 1_{0}$-FePt for polarization of magnetic films}

Yannick Dusch, ${ }^{\text {a) }}$ Nicolas Tiercelin, Alexey Klimov, ${ }^{\text {b) }}$ Vasyl Rudenko, Yury Ignatov, ${ }^{\text {b) }}$ Sami Hage-Ali, Philippe Pernod, and Vladimir Preobrazhenskyc)

International Associated Laboratory LEMAC: IEMN, UMR CNRS 8520, PRES Lille Nord de France, ECLille, 59651

Villeneuve d'Ascq, France

In various micro and nanosystems applications comprising magnetic films, the polarizing field still needs to be integrated. We hereby present a solution for the self biasing of magnetic films using micropatterned permanent magnets. Micromagnetic simulations were used as a designing and optimization tool to create a biasing structure. The samples were fabricated with varying geometric parameters using classical silicon microfabrication techniques. Nanostructured $\mathrm{TbCo} / \mathrm{FeCo}$ magnetostrictive thin films were sputtered over coercive FePt filled trenches etched in silicon. Magnetic and magneto-elastic characterizations confirmed the numerical simulations. In particular, nonlinear actuation of a self-biased magnetostrictive cantilever has been obtained at a zero external polarizing field.

\section{INTRODUCTION}

The polarization of free magnetic layers in micro- and nano-electro-mechanical-systems (MEMS and NEMS) can be obtained by several means depending upon the requirements of the application. Macroscopic magnets can be used in research or industrial environments but prevent any size reduction of the systems. Spintronic devices or NEMS can benefit from thin exchange coupled layers. Nevertheless, due to the short length of the interactions, such nanostructures are hard to implement for thick active layers in the $100 \mathrm{~nm}-1 \mu \mathrm{m}$ range. The use of permanent magnet-induced boundary fi $\mathrm{s}$ has also been investigated using $\mathrm{SmCo}^{1}$ or $\mathrm{CoPt}^{2}$ and was proven to be efficient for biasing magnetoresistive layers up to $200 \mathrm{~nm}$ and several hundred oersteds. In any case, the typical length of the system along the biasing direction did not exceed a few hundredmicrons.

We hereby propose the use of micropatterned $L 1_{0}-\mathrm{FePt}$ sputtered films to polarize magnetic thin films with larger inplane dimensions. In this study, we used such structures to induce a spin reorientation transition (SRT) in giant magne tostrictive nanostructured thin films for applications in

MEMS and NEMS. Indeed, it was shown that nanostructured giant magnetostrictive films under field induced SRT can be made highly sensitive to magnetic excitations and exhibit strong nonlinear behavior. ${ }^{3}$ This feature of the SRT conditions opens the route toward, for example, original magneto- electric microsensors, microactuators, or $\mathrm{RF}$ devices. ${ }^{4}$ In particular, the magnetoelectric effect is dramatically enhanced near the SRT in composite magnetostrictive/piezo- electric layered structures. ${ }^{5}$ Such a critical state can only be obtained when the bias field $H_{\text {Bias }}$ directed along the hard

\footnotetext{
a) Author to whom correspondence should be addressed. Electronic mail: yannick.dusch@centraliens-lille.org.

${ }^{b}$ International Associated Laboratory LEMAC: V. A. Kotelnikov Institute of Radioengineering and Electronics, 125009 Moscow, Russia.

${ }^{c}$ International Associated Laboratory LEMAC: Wave Research Center, GPI RAS, 38 Vavilov str., Moscow, 119991, Russia.
}

axis of magnetization (HA) reaches the anisotropy field $H_{a}$ of the layer.

\section{NUMERICAL SIMULATIONS}

As shown in Fig. 1, the structure consists of parallel FePt trenches etched in a silicon substrate on top of which a Ta spacer and a $[\mathrm{TbCo}(5 \mathrm{~nm}) / \mathrm{FeCo}(5 \mathrm{~nm})]^{*}$ n multilayer are deposited. The multilayer exhibits uniaxial magnetic anisotropy. The easy axis of magnetization (EA) is obtained in the multilayer thanks to sputtering under a magnetic field. Magnetostatic simulations of the flux density show that alternate polarized areas are created in the active layer due to the coupled or isolated flux closure (Fig. 2). This leads to a striped magnetic structure in the active layer (parts 1 and 2, as shown in Fig. 1). The shape of the global hysteresis curve of the active layer along the HA can be evaluated by super- posing the two shifted curves of the stripes, as illustrated in Fig. 3(a). Both parts exhibit a Stoner-Wohlfarth like rotation of magnetization.

Using the open source micromagnetic simulation software Magpar, ${ }^{6}$ we investigated the effects of several biasing structures on a magnetic thin film with in-plane uniaxial anisotropy. It appears that the structure presented above offers a good trade-off between the obtained polarization along the HA and technological simplicity. Figure 3(b) presents a typical result of the numerical simulations, clearly showing the striped pattern of magnetization on top of the active layer.

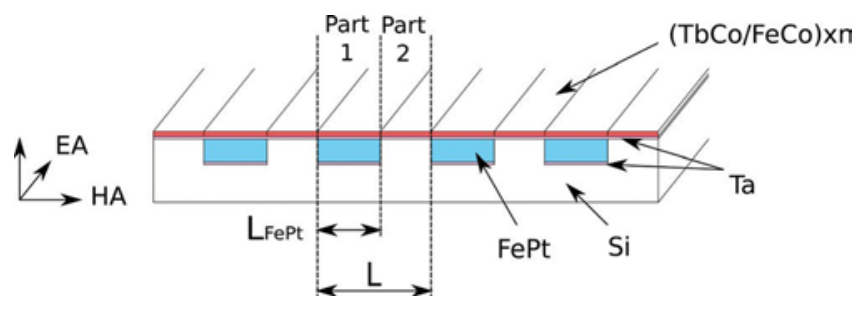

FIG. 1. Schematic view of the biasing structure. 


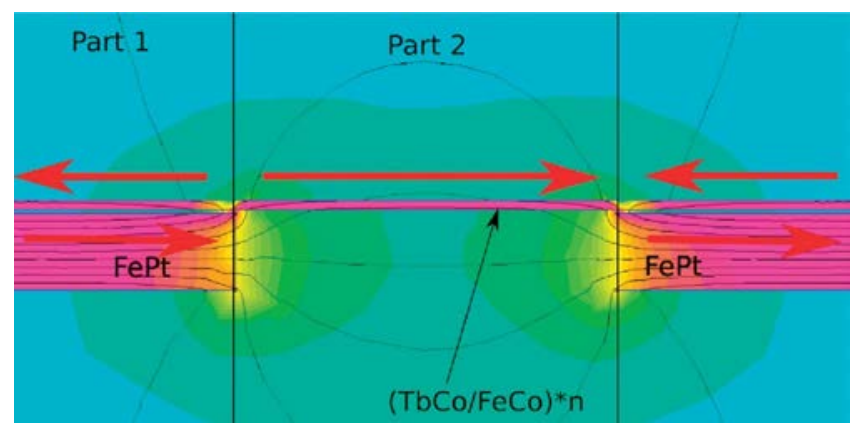

FIG. 2. Simulated flux lines [FEMM (Ref. 7). Irregularities in the flux lines are due to simulation artifacts]. Arrows indicate the main direction of magnetization.

Magpar has then been used to carry out numerous simulations with varying geometric parameters (FePt/silicon width ratio, trench width, FePt thickness, Ta gap thickness) to optimize the strength and the homogeneity of the bias field.

\section{EXPERIMENT}

Filled trenches of hard magnetic material have already been realized with SmCo and $\mathrm{NdFeB}$, but the process involved a critical phase of chemical-mechanicalplanarization. ${ }^{8}$ We present here a simpler process for FePt, based on lift-off.

Prototypes were realized on Si wafers by the usual microfabrication techniques in a cleanroom environment. Silicon is first etched through an aluminum mask by means of deep reactive ion etching (DRIE) down to $2 \mu \mathrm{m}$. A $100 \mathrm{~nm}$ Ta adhesive layer as well as a thick (2 $\mu \mathrm{m})$ FePt layer are then deposited by RF sputtering. Lift-off is used to remove the excess material. FePt is obtained by the RF sputtering of a composite target under an Ar flow at 5 x $10^{-3}$ mbar. Sputtering is decomposed into 15 min runs followed by a rest period of $4 \mathrm{~min}$ to prevent the detrimental effects of temperature, such as internal stress. A $100 \mathrm{~nm}$ Ta nonmagnetic spacer (also used to prevent oxidation of FePt during annealing) is added on top of the structure before annealing at $750^{\circ} \mathrm{C}$ during 30 min under a secondary vacuum ( $\left.1 \times 10^{-6} \mathrm{mbar}\right)$ to obtain the $L 1_{0}$ phase of FePt. This process led to a coercive field of $H_{c}=1 \mathrm{~T}$. The nanostructured active layer is deposited by RF sputtering under a homogeneous magnetic field (obtained by permanent magnets placed on the substrate holder). Alternating the sputtering of TbCo and FeCo composite targets led to 25 exchange- coupled $10 \mathrm{~nm}$ bilayers (250

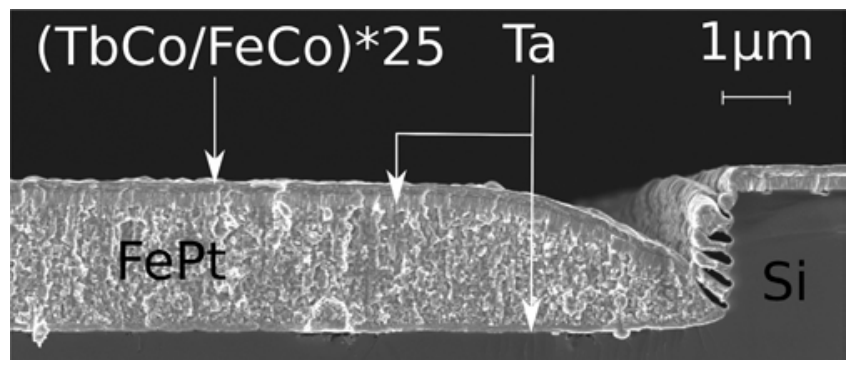

FIG. 4. SEM view of the processed microstructure.

$\mathrm{nm}$ thick). FePt is finally polarized along HA. A typical result of this process is shown on Fig. 4. The vertical structure at the interface between FePt and $\mathrm{Si}$ is due to DRIE combined with masking effects.

Two sets of samples have been fabricated: 25 samples with centimeter dimensions on a 3 in wafer with different geometric parameters $(L=20 ; 50 ; 100 ; 200$; and $400 \mu \mathrm{m}$ and $L_{\mathrm{FePt}}=L=0: 7 ; 0: 6 ; 0: 5 ; 0: 4$; and $0: 3$ ) and 5 samples fabricated on a thin $(150 \mu \mathrm{m}) 2$ in silicon wafer to obtain centimeter- sized cantilevers for magnetoelastic characterizations.

\section{RESULTS AND DISCUSSION}

\section{A. Magnetic characterizations}

First measurements were made using a vibrating sample magnetometer (VSM) (ADE EV9 Series) with sub- $\mu m$ resolution. Figure 5 presents VSM measurements of the magnetization of a sample with the following characteristics: $L=50 \mu \mathrm{m}, L_{\mathrm{FePt}}=25 \mu \mathrm{m}$, FePt thickness $e_{\mathrm{FePt}}=2 \mu \mathrm{m}$, Ta spacer thickness $e_{\text {spacer }}=100 \mathrm{~nm}$, active layer thickness $e=250 \mathrm{~nm}$. The contribution of FePt has been removed beforehand on the curve. As shown in Fig. 5, the global hysteresis curve $M\left(H_{\text {ext }}\right)$ of the biased active layer along the HA is composed of two shifted contributions (compared to the unbiased reference), each accounting for half of the magnetic stripes. Both shifts are almost symmetrical with a bias field of approximately 500 Oe. Since in some cases slope changes are difficult to observe, we also used the derivatives of the hysteresis curves which are also presented in Fig. 5.

Longitudinal magneto-optic Kerr effect (LMOKE) measurements have been carried out to investigate the magnetic microstructure on the surface of the nanostructured active layer and validate the interpretation of the VSM measurements. Due to the size limitations of the experimental setup measurements could not be carried out on every

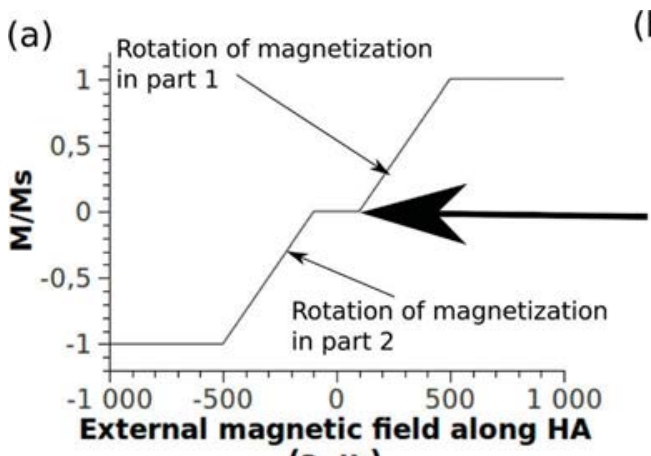

(a. u.) (b) ;

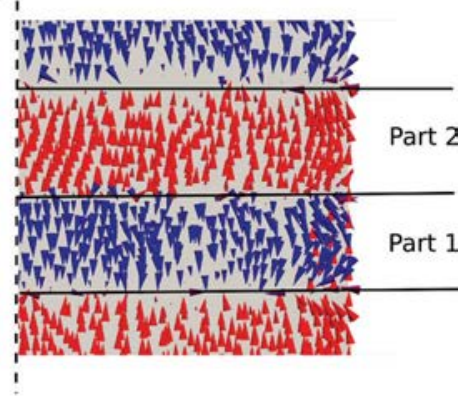

FIG. 3. (a) Schematic illustration of the hysteresis curve of the active layer when the polarizing field is superior to the anisotropy field (b) Top view of the simulated active layer with $H_{\mathrm{ext}}=0 \mathrm{Oe}, L_{\mathrm{FePt}}=25$ $\mu \mathrm{m}$ and $L=50 \mu \mathrm{m}$. Arrows indicate magnetization. 

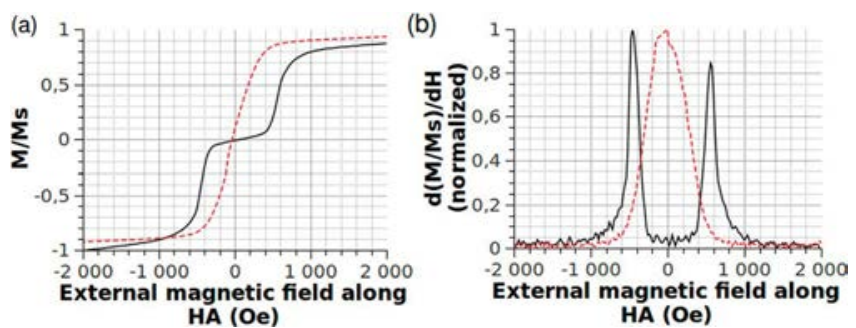

FIG.5. Hysteresis curve (a) and derivative of the hysteresis curve (b) of the active layer (Solid: biased, dashed: unbiased).

sample. Nevertheless, the results obtained for $L=100 \mu \mathrm{m}$ and $L_{\mathrm{FePt}}=50 \mu \mathrm{m}$ confirmed our interpretations. The hysteresis curves for each part of the magnetic structure can be seen in Fig. 6. As expected, when the FePt is magnetized, two adjacent stripes (namely part 1 and part 2) show opposite offsets along the HA. Here, the offsets are, respectively, 100 Oe and 200 Oe.

\section{B. Magneto-elastic characterizations}

Magneto-elastic characterizations have been carried out by a laser deflection technique on a centimeter sized silicon cantilever on top of which the biasing structure has been realized. The cantilever is placed inside the core of a driving coil producing a dynamic magnetic field along the EA of the active layer. The cantilever is then placed under a homogeneous static magnetic field created by an electromagnet along the HA. The flexural or torsional vibration amplitude of the free tip is measured by the deflection of a laser beam on a 2D position sensing diode. As predicted by theory, ${ }^{9}$ due to giant nonlinearity near the SRT, subharmonic magnetic excitation at $f_{r}=2$ with $f_{r}$ a resonant frequency of the cantilever, results in an amplified mechanical vibration at $f_{r}$ near the SRT point $\left(H_{\text {Bias }} \approx H_{a}\right)$. Figure 7 shows the results for a $2 \mathrm{~cm}$ x 0:5 cm x $150 \mu \mathrm{m}$ cantilever for the second flexural mode at $f_{r}=3124 \mathrm{~Hz}$.

As expected, nonlinear actuation of the system occurs for shifted values of the external magnetic field, compared to an unbiased sample and even exists without any external field, as the bias field is close to the anisotropy field $H_{a}=300$ Oe.
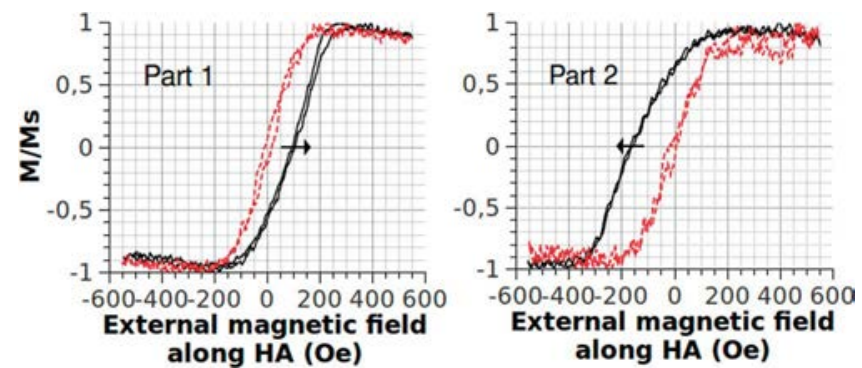

FIG. 6. MOKE measurements of a biased sample $(L=100 \mu \mathrm{m}$, $\left.L_{\mathrm{FePt}}=50 \mu \mathrm{m}\right)$ (Solid line: biased, dashed line: unbiased).

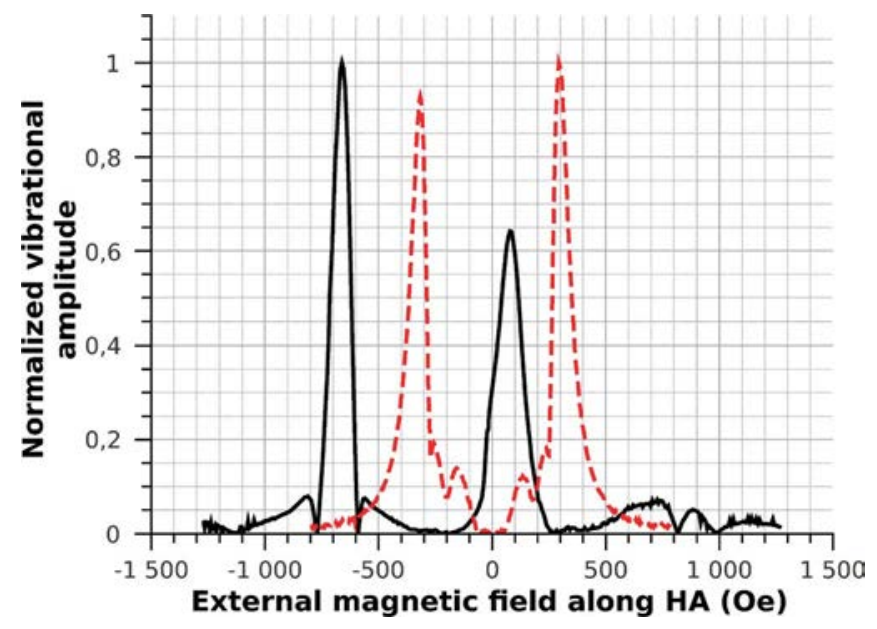

FIG. 7. Magnetoelastic measurement (Solid line: biased, dashed line: unbiased).

\section{CONCLUSIONS}

In this paper, we have proposed an integrated permanent magnet based microstructure for the polarization of magnetic thin films. Micromagnetic simulations proved efficient as a sizing and optimization tool for such structures. Several samples have been successfully fabricated due to a MEMScom- pliant process. In the case of a giant magnetostrictive thin film, integrated VSM along with local LMOKE magnetic characterizations confirmed the numerical studies. Moreover, magnetoelastic measurements showed that because of the integrated polarization, subharmonic actuation of a cantilever could be obtained without any external static field. These results pave the way for the development and the integration of innovative and multifunctional micro-magneto-mechanical- and micromagneto-electro-mechanical-systems.

\section{ACKNOWLEDGMENTS}

Y. Dusch's Ph.D. Thesis is funded by the Direction Générale de l'Armement (DGA-France). This work is part of the French ANR Project NAMAMIS.

${ }^{1}$ V. Neu, A. Anane, S. Wirth, P. Xiong, S. A. Shaheen, and F. J. Cadieu, J. Appl. Phys. 87, 5350 (2000).

${ }^{2}$ M. Kitada, Y. Kamo, H. Tanabe, H. Tsuchiya, and K. Momata, J. Appl. Phys. 58, 1667 (1985).

${ }^{3}$ N. Tiercelin, J. B. Youssef, V. Preobrazhensky, P. Pernod, and H. L. Gall, J. Magn. Magn. Mater. 249, 519 (2002).

${ }^{4}$ A. Klimov, Yu. Ignatov, N. Tiercelin, V. Preobrazhensky, P. Pernod, and S. Nikitov, J. Appl. Phys. 107, 093916 (2010).

${ }^{5}$ N. Tiercelin, A. Talbi, V. Preobrazhensky, P. Pernod, V. Mortet, K. Haenen, and A. Soltani, Appl. Phys. Lett. 93, 162902 (2008).

${ }^{6}$ W. Scholz, J. Fidler, T. Schrefl, D. Suess, R. Dittrich, H. Forster, and V. Tsiantos, Comput. Mater. Sci. 28, 366 (2003).

${ }^{7}$ D. C. Meeker, Finite Element Method Magnetics, Version 4.0.1 (03Dec2006 Build), http://www.femm.info

${ }^{8}$ A. Walther, C. Marcoux, B. Desloges, R. Grechishkin, D. Givord, N. M. Dempsey, J. Magn. Magn. Mater. 321, 590 (2009).

${ }^{9}$ N. Tiercelin, P. Pernod, V. Preobrazhensky, H. Le Gall, J. Ben Youssef, Ultrasonics 38, 64 (2000). 